\title{
BMJ Open Trends in high deductible health plan enrolment and spending among commercially insured members with and without chronic conditions: a Natural Experiment for Translation in Diabetes (NEXT-D2) Study
}

Laura F Garabedian (D) , Fang Zhang, Robert LeCates, Jamie Wallace, Dennis Ross-Degnan, James F Wharam

To cite: Garabedian LF, Zhang F, LeCates $\mathrm{R}$, et al. Trends in high deductible health plan enrolment and spending among commercially insured members with and without chronic conditions: a Natural Experiment for Translation in Diabetes (NEXT-D2) Study. BMJ Open 2021;11:e044198. doi:10.1136/ bmjopen-2020-044198

- Prepublication history and supplemental material for this paper is available online. To view these files, please visit the journal online (http://dx.doi. org/10.1136/bmjopen-2020044198).

Received 01 September 2020 Accepted 17 June 2021

Check for updates

(C) Author(s) (or their employer(s)) 2021. Re-use permitted under CC BY-NC. No commercial re-use. See rights and permissions. Published by BMJ.

Division of Health Policy and Insurance Research, Department of Population Medicine, Harvard Medical School and Harvard Pilgrim Health Care Institute, Boston, Massachusetts, USA

Correspondence to Dr Laura F Garabedian; laura.garabedian@post.harvard. edu

\section{ABSTRACT}

Objectives To examine trends in high deductible health plan (HDHP) enrolment among members with diabetes and cardiovascular disease (CVD) compared with healthy members and compare out-of-pocket (OOP) and total spending for members with chronic conditions in HDHPs versus low deductible plans.

Design Descriptive study with time trends.

Setting A large national commercial insurance database. Participants 1.2 million members with diabetes, 4.5 million members with CVD (without diabetes) and 18 million healthy members (defined by a low comorbidity score) under the age of 65 years and insured between 2005 and 2013.

Outcome measures Percentage of members in an HDHP (ie, annual deductible $\geq \$ 1000$ ) by year, annual mean 00P and total spending, adjusted for member sociodemographic and employer characteristics.

Results Enrolment in HDHPs among members in all disease categories increased by 5 percentage points a year and was over $50 \%$ by 2013 . On average, over the study period, HDHP enrolment among members with diabetes and CVD was 2.84 (95\% Cl: 2.78 to 2.90) and 2.02 (95\% Cl: 1.98 to 2.05) percentage points lower, respectively, than among healthy members. HDHP members with diabetes, CVD and low morbidity had higher annual 00P costs ( $\$ 636$ (95\% Cl: 630 to 642), $\$ 539$ (95\% Cl: 537 to 542$)$ and $\$ 113$ (95\% Cl: 112 to 113)) and lower total costs ( $\$ 529$ (95\% Cl: -597 to $-461),-\$ 364(95 \% \mathrm{Cl}:-385$ to -342$)$ and $-\$ 79(95 \%$ $\mathrm{Cl}:-81$ to -76$))$, respectively, than corresponding low deductible members when averaged over the study period. Members with chronic diseases had yearly OOP expenditures that were five to seven times higher than healthier members.

Conclusion High HDHP enrolment coupled with the high OOP costs associated with HDHPs may be particularly detrimental to the financial well-being of people with diabetes and CVD, who have more healthcare needs than healthier populations.
Strengths and limitations of this study

- This is the first study to compare enrolment in high deductible health plans between members with chronic diseases (ie, diabetes and cardiovascular disease) and healthy members.

- This study uses 9 years of claims data from a large, national health insurer in the USA.

- The study also examines out-of-pocket and total costs between members in high deductible and low deductible plans.

- The study is descriptive and we cannot infer causal relationships.

- The data do not include insurance premium information, so we cannot assess the full financial burden on members.

\section{INTRODUCTION}

Recent research suggests that high deductible health plans (HDHPs), which provide incentives for patients to manage their own healthcare costs, are associated with concerning impacts on patients with chronic conditions. However, little is known about trends in HDHP enrolment among patients with chronic conditions versus healthier populations and the associated economic burden.

To reduce healthcare costs and monthly premiums, an increasing number of employers offer HDHPs. These arrangements provide incentives for patients to use select high-value services and reduce healthcare costs through inexpensive preventive care and higher annual deductibles. ${ }^{1}$ In 2019, $82 \%$ of commercially insured Americans had an annual deductible; of these, over twothird $(69 \%)$ had a deductible of over $\$ 1000$ and over 1 in 10 (14\%) had a deductible 
over $\$ 3000 .^{2}$ HDHPs paired with a tax-preferred savings account for out-of-pocket (OOP) spending (ie, health savings accounts (HSA) or health reimbursement arrangements (HRA)) are the most rapidly growing plan type, now covering $30 \%$ of commercially insured Americans. ${ }^{2}$ HDHPs have been shown to reduce healthcare spending, but also reduce preventive care ${ }^{3}$ and cause members to delay care because of costs. ${ }^{4}$ If given a choice in plans by their employer, employees who choose HDHPs tend to be younger and healthier and more likely to live in neighbourhoods with a higher proportion of individuals of higher income, higher education and white race. ${ }^{5}$

Diabetes and cardiovascular disease (CVD) are the two most prevalent chronic illnesses in the USA. High quality of care for such conditions requires multiple office visits, tests, examinations and medications. ${ }^{6-13}$ Typically, HDHPs fully cover some preventive services and one annual preventive visit, but require full cost-sharing up to the annual deductible for all other services and often additional cost sharing (ie, coinsurance or copayment) after the deductible is met. Our Natural Experiments in Diabetes Translation 1 (NEXT-D1) study used a robust study design to examine the impact of HDHPs among members with diabetes. Studies demonstrated decreased utilisation of both appropriate and discretionary services, with concerning impacts on vulnerable populations. For example, HDHPs were associated with delays in seeking care for major macrovascular disease symptoms, diagnostic tests and procedure-based treatments, ${ }^{14}$ reductions in specialist visits, ${ }^{15}$ delayed outpatient visits for acute preventable complications ${ }^{15}$ and higher emergency department visits for acute complications among the poor. ${ }^{15}$ The negative impacts of HDHPs are consistently more pronounced in low-income HDHP members or members with an HSA-HDHP. ${ }^{15} 16$ Other studies have suggested similar impacts of HDHPs on members with CVD. $^{17}$

Despite these concerning effects, trends in HDHP enrolment and OOP burden among patients with chronic illness versus healthier patients are unknown. Our objective was to assess 2005-2013 trends in HDHP enrolment among members with diabetes and CVD in a large national insurer, compared with a cohort of healthy members. We also compared the demographics, comorbidities and trends in OOP spending and total spending of members with chronic conditions and healthier members in HDHPs (with and without savings accounts) to counterparts in low deductible plans.

\section{METHODS}

\section{Study design}

This descriptive study assessed annual trends from 2005 to 2013 and differences in HDHP enrolment between subgroups of members with chronic conditions compared with healthy members and, within disease category, assessed member-level and employer-level characteristics associated with HDHP enrolment and compared differences in OOP spending and total costs between members with HDHPs and low-deductible health plans.

\section{Data}

We used a large claims database that included approximately 55.5 million unique commercially insured members of all ages from 2005 to 2013. Members with Medicare Advantage were excluded from this study since they were not subject to comparable insurance arrangements. The data included enrolment status and all medical and pharmacy claims. We used the Johns Hopkins Adjusted Clinical Group (ACG) system (V.11.1), ${ }^{18} 19$ to assign diagnostic categories and an overall comorbidity score using claims data (ie, diagnoses, procedures and medications) from the prior 12 months. We also linked individuals to neighborhood-level socioeconomic characteristics from the 2008-2012 American Community Survey (5-year estimates at the census tract level).$^{20}$

\section{Study population}

We included members under the age of 65 years with diabetes and CVD and a comparison group of healthier members. We created the three mutually exclusive categories of members based on ACG diagnostic categories: diabetes (inclusive of type 1 and type 2 diabetes) with or without CVD; CVD or risk factors (ie, lipid disorders or hypertension) without diabetes; and 'healthy' (defined as ACG morbidity score $\leq 1$ and excluding members with a diabetes or CVD diagnosis). We included members with at least 12 months of continuous enrolment covered by employers insuring 10 or more members (for whom we could reliably assess HDHP status). We used the ACG diagnosis flag (or score) from the last month of each member's 12-month enrolment period (ie, 'anniversary month'). Measures calculated over each 12-month enrolment period were assigned to the calendar year of each anniversary month.

\section{Outcomes and covariates}

For each annual employer enrolment period, we classified members as being enrolled in an HDHP or nonHDHP plan on the anniversary month. We used actual or imputed deductible levels; the imputations were based on adding actual deductible payments per person per benefit year at the employer then assigning a deductible level to that employer using a regression model that included the summed deductible levels of all enrollees and other employer characteristics (online supplemental appendix 1). Using a common convention, we defined HDHPs as plans with a deductible level $\geq \$ 1000$ and low deductible plans as plans with a deductible level $\leq \$ 500$. Within HDHPs, we examined two levels of HDHP (ie, \$1000-\$2499 and $\geq \$ 2500$ ) and identified HDHP members with a savings account plan (ie, HSA or HRA) using flags provided by the data vendor.

For member demographics, we examined age; sex; region (ie, Midwest, Northeast, South and West); neighborhood-level income (ie, low poverty, low-medium 
poverty, high-medium poverty and high poverty); education (ie, low, low-medium, high-medium and high education level) and race/ethnicity (ie, white, non-Hispanic vs all other races/ethnicities) using ACS categories (online supplemental appendix 2). As a measure of comorbidity, we included the ACG score, measured as a continuous variable. We also measured two employer-level variables: self-insured versus fully-insured status and employer size (ie, 10-99, 100-999 and $\geq 1000$ employees). All covariates were measured on the anniversary month.

We calculated the annual OOP expenditures of members, which included all cost sharing (ie, deductibles, copayments and coinsurance), but not premium payments, adjusted for inflation to 2015 USD values using the Consumer Price Index for medical care, and total medical expenditure (ie, insurer's allowed amount inclusive of OOP costs, commonly referred to as 'total cost') using a vendor-provided field that standardises claimslevel prices across geography and time, which is inflationadjusted to 2015 .

Using employer-level data, we determined which members had a choice of an HDHP or a plan with a lower deductible from their employer. As a secondary analysis, we examined HDHP enrolment in the subset of members with employer-level plan choice.

\section{Statistical analysis}

We first generated descriptive statistics of demographic and employer characteristics for HDHP and low deductible members in each of the three disease categories. We then used generalised estimating equations (GEE), applying the robust sandwich estimator and assuming an exchangeable working correlation structure to account for member-level clustering (since a member could contribute to the database for multiple years), with marginal models to assess all outcomes. ${ }^{21}$ We used average adjusted predictions ${ }^{22}$ to examine member-level predictors of being in an HDHP within each disease category, controlling for study year. Predictors in the model included the member-level and employer-level characteristics mentioned above (ie, age, sex, region, income, education, race/ethnicity, ACG score, self-insured status, employer size and study year). We also used average adjusted prediction models to estimate annual percentage of members enrolled in an HDHP and trends (ie, slope) in HDHP enrolment for each disease category, controlling for the same variables as the enrolment prediction model. We calculated average marginal effects on the GEE models ${ }^{22}$ to estimate the average difference in the percentage of members enrolled in an HDHP over the study period between each chronic disease group and healthier members, controlling for the same variables as the prediction model, except for ACG score (which is highly collinear with our disease categories).

Within each disease category, we used GEE models and adjusted prediction at the means, ${ }^{22}$ controlling for the same variables as the HDHP enrolment prediction model, to examine the adjusted annual OOP and total costs for members with high and low deductible plans and the trends in costs over time for each disease category. Within each disease category, we used marginal effects at the means to estimate the absolute and relative differences in OOP and total costs between HDHP and low deductible health plan (LDHP) members. For each study year, we also calculated the average percent of total expenditure that HDHP members paid OOP (based on adjusted values), by disease category. And, within each disease category, we examined average comorbidity (ie, ACG) score over the study period for members in high versus low deductible plans.

In the models to assess percentage of members enrolled in an HDHP and predictors of HDHP enrolment, the denominator was all members in that disease category. The analyses that examined OOP and total costs compared members in HDHP $(\geq \$ 1000)$ to members in low deductible plans $(\leq \$ 500)$ and therefore excluded members with deductibles of \$501-\$999.

All analyses were performed in SAS Studio V.3.7 or STATA V.15.

\section{Patient and public involvement}

Patients or the public were not involved in the design, conduct, reporting, or dissemination plans of our research.

\section{RESULTS}

Our sample included three mutually exclusive disease categories: 1.2 million unique members (2.6 million member-years) with diabetes, 4.5 million unique members (9.4 million member-years) with CVD and risk factors (without diabetes) and 18 million unique healthy members (40.4 million member-years). Online supplemental appendix 3 includes demographic and employer characteristics for members in HDHP and LDHP.

\section{Predictors of HDHP enrolment within disease category}

In the predicted probability models (table 1), HDHP enrolment among members with diabetes and CVD and healthy members was statistically significantly higher for members with the following characteristics: higher income; white, non-Hispanic race/ethnicity; lower comorbidity score; living in the West, Midwest or South (compared with the Northeast); being insured through a fully-insured (vs self-insured) employer; and working for a smaller employer. Age had mixed results across disease categories. Across all disease categories, the largest absolute predictors of HDHP status were region, fully-insured employer status and smaller employer size. For instance, on average, over the study period, $49.30 \%$ (95\% CI: $49.13 \%$ to $49.47 \%$ ) of members with diabetes insured through a smaller employer (ie, 10-99 enrollees) were enrolled in an HDHP compared with $23.83 \%$ (95\% CI: $23.72 \%$ to $23.94 \%$ ) of members with diabetes insured through a large employer (ie, $\geq 1000$ enrollees). 


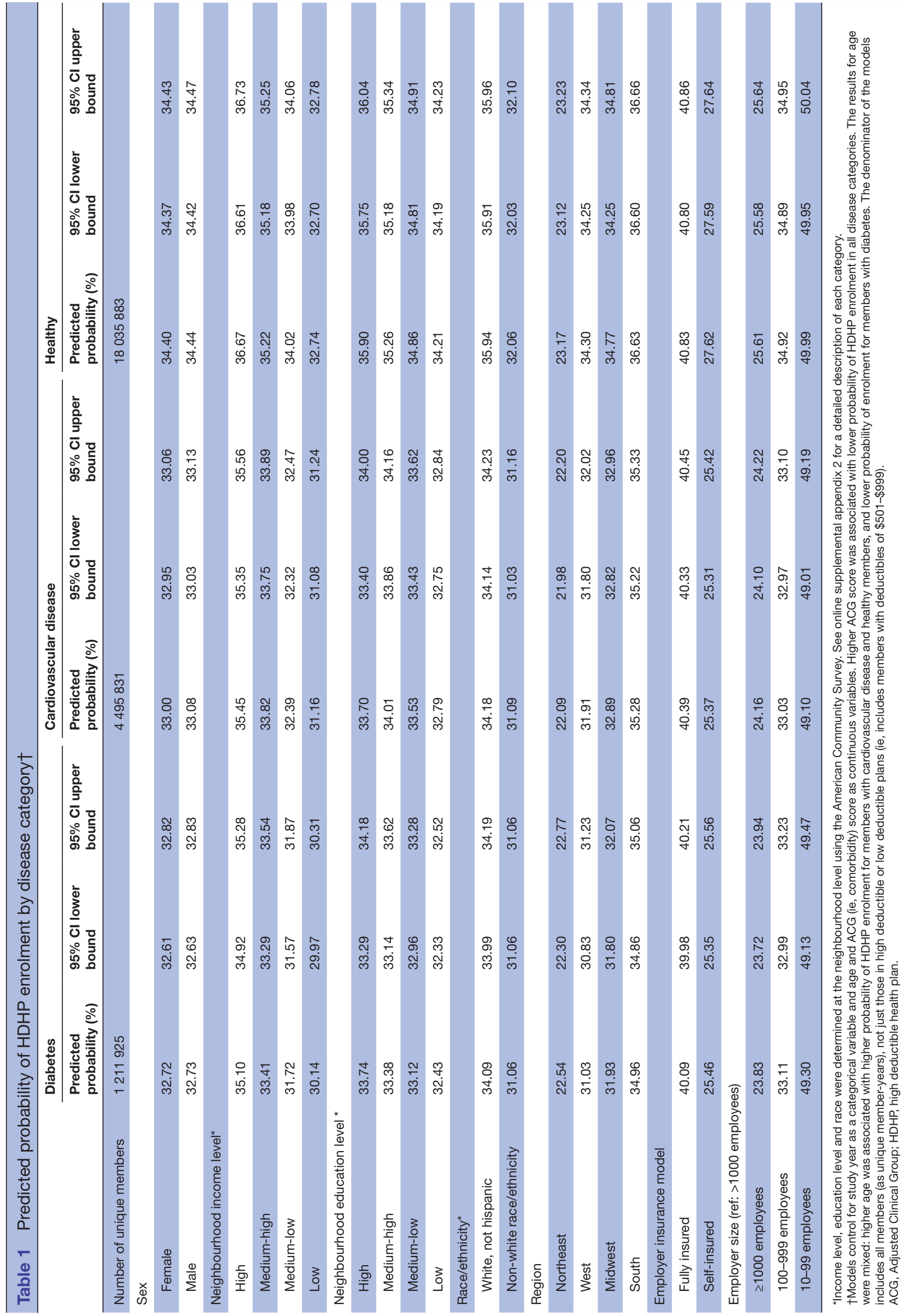




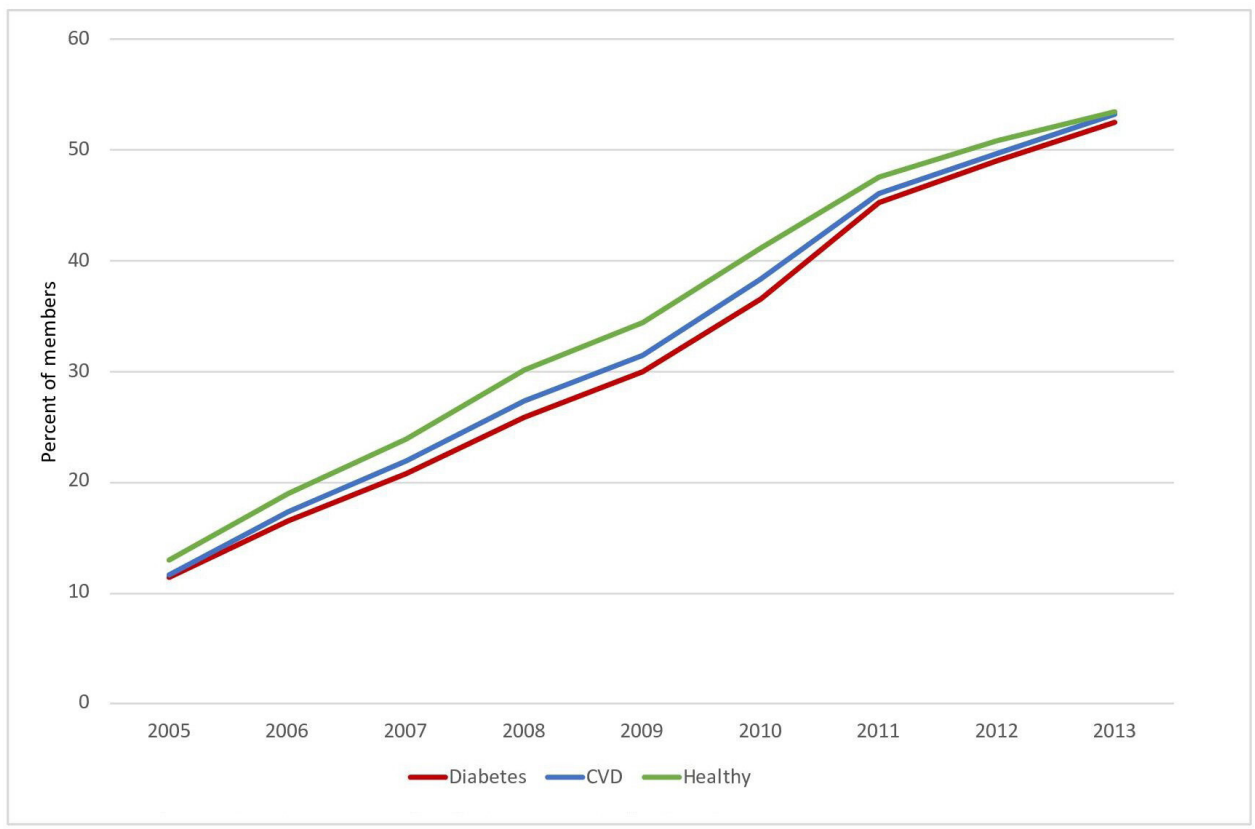

Figure 1 Percentage of members enrolled in an HDHP, by disease category (adjusted). The denominator includes all members, not just those in high deductible or low deductible plans (ie, includes members with deductibles of \$501-\$999). Estimates are adjusted for variables in table 1 using marginal models and average adjusted predictions. CVD, cardiovascular disease; HDHP, high deductible health plan.

\section{Percentage of members enrolled in an HDHP over time}

Enrolment in HDHPs increased markedly over the study period for all disease categories, increasing by approximately 5 percentage points per year across all groups when adjusting for the variables in table 1 . HDHP enrolment increased by 5.29 percentage points (95\% CI: 5.27 to 5.31 ), 5.27 percentage points (95\% CI: 5.26 to 5.28 ), and 5.11 percentage points (95\% CI: 5.10 to 5.12 ) every year for members with diabetes, CVD and healthy members, respectively, over the study period. Members with chronic diseases had lower levels of HDHP enrolment than healthier members throughout the entire study period (figure 1). On average, over the study period, HDHP enrolment among members with diabetes and CVD was 2.84 (95\% CI: 2.78 to 2.90 ) and 2.02 (95\% CI: 1.98 to 2.05) percentage points lower, respectively, than among healthy members. However, by the end of the study period, over half of the members in each disease category were in an HDHP. In 2013, 53.43\% (95\% CI: $53.39 \%$ to $53.47 \%$ ) of healthy members were in an HDHP compared with $52.48 \%$ (95\% CI: $52.33 \%$ to $52.63 \%$ ) of members with diabetes and $53.21 \%$ (95\% CI: $53.12 \%$ to $53.29 \%$ ) of members with CVD in the adjusted models. While nonaccount HDHPs were the most common HDHP type for HDHP members in all three disease categories (online supplemental appendix 4a), the higher percentage of enrolment in HDHPs among healthy members, compared with members with chronic diseases, was driven by higher enrolment in HSA-eligible HDHPs (online supplemental appendix 4b). The percentage of members enrolled in a very high deductible health plan $(\geq \$ 2500)$ increased over the study period for all disease categories, from less than
$1 \%$ in 2005 to $14 \%-15 \%$ in 2015 (online supplemental appendix 5). In the last 3 years of the study period, the percentage of members in an HDHP with a deductible between $\$ 1000$ and $\$ 2499$ remained relatively flat, and the increase observed in HDHP plan enrolment overall was driven by enrolment in very high deductible health plans.

During the study period, the percent of members with an employer that offered both HDHPs and a lower deductible plans increased from $10 \%-11 \%$ in 2005 to $33 \%-34 \%$ in 2013 in all disease areas (online supplemental appendix 6). The percentage of members with employer-level plan choice that enrolled in an HDHP increased over the study period, but was 12-13 percentage points lower than the percentage of all members (ie, with and without plan choice). In 2013, among members with employer-level plan choice, $41.25 \%$ (95\% CI: $41.18 \%$ to $41.32 \%$ ) of healthy members, $39.10 \%$ (95\% CI: $38.83 \%$ to $39.37 \%$ ) of members with diabetes and $40.58 \%(95 \%$ CI: $40.43 \%$ to $40.73 \%$ ) with CVD were in an HDHP. On average, over the study period, members with plan choice who had a chronic disease were 4-5 percentage points less likely than healthier members with plan choice to enrol in an HDHP.

\section{Out-of-pocket costs}

For all disease categories, HDHP members had higher OOP costs than low deductible plan members (figure 2). The differences in OOP costs between HDHP and low deductible plans were, on average over the study period, \$636 (95\% CI: 630 to 642), \$539 (95\% CI: 537 to 542) and $\$ 113$ (95\% CI: 112 to 113) for members with 


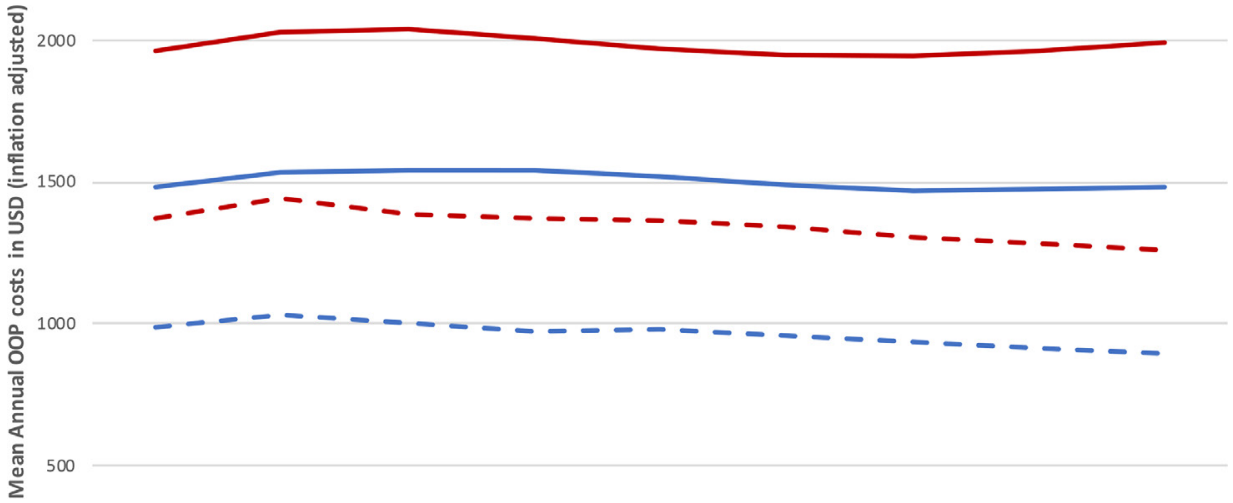

diabetes, CVD and healthy members, respectively. In relative terms, compared with LDHP members, average OOP costs for HDHP members were $47.26 \%$ (95\% CI: $46.73 \%$ to $47.78 \%), 56.03 \%(55.71 \%$ to $56.63 \%)$ and $70.41 \%$ (95\% CI: $70.13 \%$ to $70.69 \%$ ) higher, respectively. Inflation-adjusted OOP costs decreased slightly over time for both LDHP and HDHP members in all three disease categories, with a steeper decline observed among LDHP members. OOP costs for low deductible plan members decreased, on average, by $\$ 20$ (95\% CI: 19 to 21$), \$ 17$ (95\% CI: 17 to 18 ) and $\$ 6$ (95\% CI: 6 to 6 ) every year for members with diabetes, CVD and healthy members, respectively, with corresponding decreases for HDHP members of $\$ 6$ (95\% CI: 4 to 8 ), $\$ 10$ (95\% CI: 9 to 11 ) and $\$ 3$ (95\% CI: 3 to 4 ) every year. Across all disease categories, members in HRA-eligible and HSA-eligible HDHPs had higher OOP costs than non-account HDHPs (online supplemental appendix 7).

\section{Total costs}

For all disease categories, HDHP members had lower total costs than low deductible plan members (figure 3). The differences in total costs between HDHP and low deductible plans were, on average over the study period, $-\$ 529$ (95\% CI: -597 to -461$),-\$ 364$ (95\% CI: -385 to -342 ) and $-\$ 79$ (95\% CI: -81 to -76$)$ for members with diabetes, CVD and healthy members, respectively. In relative terms, compared with low deductible members, average total costs for HDHP members were $5.35 \%$ (95\% CI: $4.66 \%$ to $6.04 \%$ ), $5.60 \%$ (95\% CI: $5.27 \%$ to $5.93 \%$ ) and $9.05 \%$ (95\% CI: $8.77 \%$ to $9.33 \%$ ) lower for members with diabetes, CVD and healthy members, respectively. Our measure of total costs decreased over the study period for both HDHP and LDHP members in all three disease categories, with a steeper decrease observed among HDHP members. Total costs for low deductible plan members decreased, on average, by $\$ 248$ (95\% CI: 229 to 267$), \$ 449$ (95\% CI: 434 to 464$)$ and $\$ 21$ (95\% CI: 20 to 22) every year for members with diabetes, CVD and healthy members, respectively, with corresponding decreases for HDHP members of $\$ 348$ (95\% CI: 318 to 379), \$567 (95\% CI: 544 to 589 ) and \$28 (95\% CI: 26 to 29) every year, respectively. Members with chronic diseases in HRA-eligible and HSA-eligible HDHPs had consistently higher total costs than non-account HDHPs (online supplemental appendix 8).

Members with chronic diseases in both high and low deductible plans had higher OOP costs and total expenditures than healthy members in similar plans (figures 2 and 3). While the OOP share of total expenditure of HDHP members was lower for members with chronic diseases (ie, on average over the study period, OOP share was $21 \%$ of total expenditure for members with diabetes, $25 \%$ for members with CVD and $35 \%$ for healthy members), HDHP members with chronic diseases had yearly OOP expenditures that were five (for CVD members) to seven (for diabetes members) times higher than healthy HDHP members (online supplemental appendix 9).

Within each disease category, HDHP members had lower comorbidity scores than low deductible plan members and the comorbidity scores remained relatively stable over time (online supplemental appendix 10). 

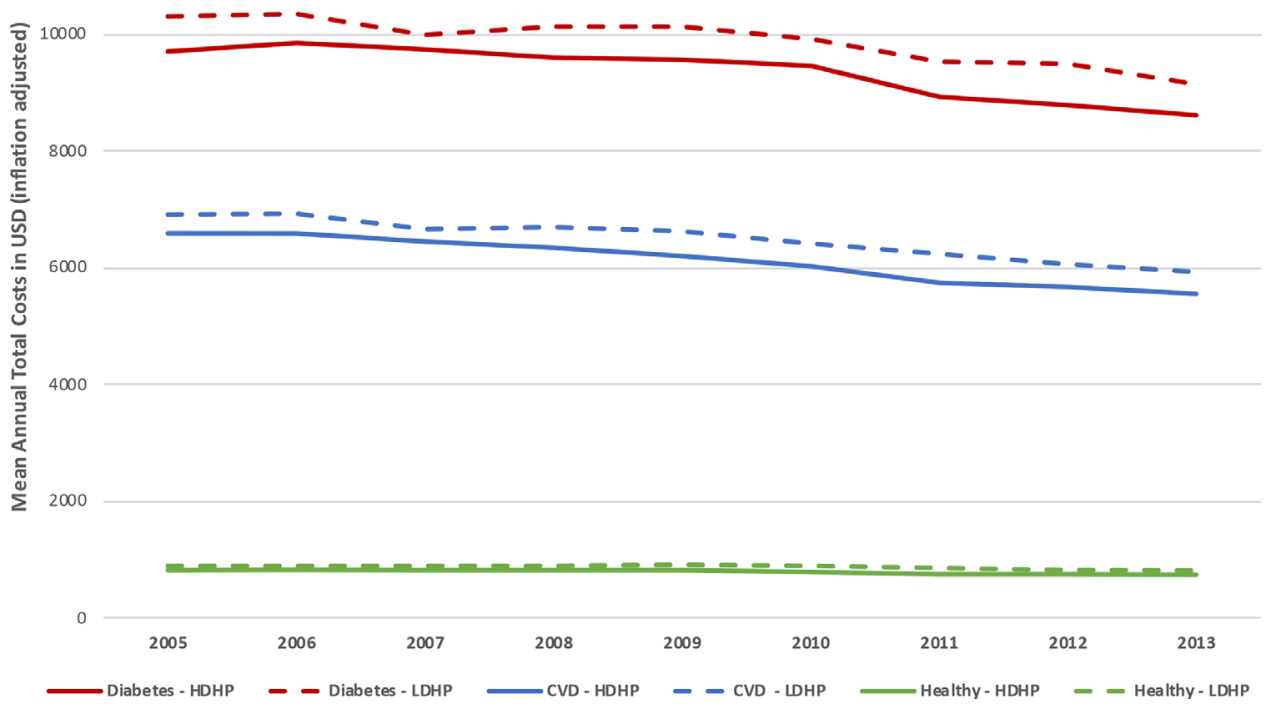

Figure 3 Mean annual total costs by disease category, HDHP versus LDHP (adjusted). Total cost estimates are adjusted for variables in table 1 using marginal models and adjusted prediction at the means. CVD, cardiovascular disease; HDHP, high deductible health plan (ie, annual deductible $\geq \$ 1000$ ); LDHP, low deductible health plan (ie, annual deductible $\leq \$ 500$ ).

\section{DISCUSSION}

HDHP enrolment increased rapidly among both chronically ill and healthy commercially insured individuals from 2005 to 2013. Members with diabetes and CVD had slightly lower levels of HDHP enrolment than healthy members throughout the study period. However, by the end of the study period in 2013, over half $(52 \%-53 \%)$ of the members with chronic conditions and healthy members were in HDHPs. Similar to previous research, we found that members who were healthier and lived in neighbourhoods with higher income and education and a higher proportion of white, non-Hispanic individuals were more likely to be in an HDHP. ${ }^{5}$ Across all disease categories, members insured through larger and selfinsured employers were significantly less likely to be in an HDHP, suggesting that these employers may offer more generous benefit packages to their employees. Among the subset of members who were offered a choice of an HDHP or lower deductible plan from their employer, most members opted for a lower deductible health plan and members with chronic diseases were less likely to choose an HDHP than healthier members.

As expected, members with chronic diseases in both high and low deductible plans have higher OOP costs and total expenditures than healthy members in similar plans. However, the OOP cost burden was five to seven times higher for HDHP members with chronic diseases compared with healthy members in HDHPs. These findings are concerning because our previous studies have shown that HDHPs are associated with avoided or delayed care and adverse health outcomes among diabetes patients ${ }^{14-16}$ and other studies suggest similar impacts of HDHPs among members with CVD ${ }^{17}$ Higher OOP costs combined with stagnant incomes ${ }^{23}$ and increasing HDHP enrolment among patients with chronic conditions suggests increasing financial burden on this vulnerable population.

Within each disease category, members in HDHPs had higher OOP costs, but lower total medical expenditures than those in low deductible plans. Lower total medical costs among HDHP members has been demonstrated in other studies. ${ }^{3}$ The lower total costs among HDHP members in our study could indicate that HDHP members are different or healthier than low deductible members in ways not captured by our adjusting covariates. However, it could also reflect less utilisation among HDHP members in response to increased cost sharing. We observed decreasing trends in total costs over time for both HDHP and LDHP members across all disease categories. Since our measure of total cost is based on standardised prices over time, and expenditure is pricexquantity, this suggests that utilisation is decreasing over time among all members. The larger downward trend in total cost among HDHP members compared with low deductible plan members suggests a greater decrease in utilisation among HDHP members. HSA-eligible and HRA-eligible HDHP members with chronic diseases consistently had higher OOP and higher total costs than members in nonaccount based HDHPs (online supplemental appendix 7), possibly because the accounts provide funds that lower barriers to utilisation and reduce the effective OOP cost of care through use of pretax dollars. However, another study found that more than half of the members with HSAeligible HDHPs do not contribute money to their HSA, ${ }^{24}$ suggesting that HSA accounts may not actually reduce the OOP burden for the majority of HDHP members. Our finding that members with chronic diseases paid OOP for a lower share of total costs than healthier members 
is because members with chronic diseases have much higher total costs and many high costs members hit their deductible and OOP max limits. ${ }^{25}$

Our study has multiple limitations. The ACG codes and scores rely on the appearance of diagnoses in medical claims. There may be increased provider coding of chronic conditions over time as risk-adjustment payment became more prominent, or reduced coding among HDHP members if they were less likely to seek routine care. However, we found that ACG scores among each disease category remained relatively stable over our study period (online supplemental appendix 10) and past analyses have shown minimal reductions in outpatient visits among members who were forced to switch into an HDHP. ${ }^{15}$ Members may make healthcare decisions based on their total expected costs, which include OOP costs and premiums, minus contributions to savings accounts. While we have data on OOP costs, we do not have data on premium amounts or on employer and employee contributions to savings accounts, so total member expenditure is unknown. Increased OOP costs in HDHPs may be offset by the lower premiums or employer contributions to accounts or by increased wages. Our study includes data from large, mid-size and small employers with commercial health insurance plans offered by a large, national insurer; therefore, our study results may not be generalisable to regional plans, very small employers $(<10$ members) or members insured in the non-group market. Although we knew the exact deductible level of most smaller employers, we had to infer it from claims at large employers. However, the sensitivity and specificity of our algorithm was high and increased across employer size category, ranging from $96 \%$ to $100 \%$ (online supplemental appendix 1). We expect adjustment for the uncertainty of the imputation process would have a negligible effect on confidence bounds. We were missing neighborhood-level socioeconomic variables for a small proportion of members $(\leq 3 \%$ across the disease and deductible level categories, online supplemental appendix 3) and these members were excluded from the GEE models. Finally, since our study aimed to examine overall trends in HDHP enrolment and costs, our main analyses combined HDHP members whose employers offered only an HDHP with members who were offered a choice by their employer to enrol in an HDHP or a lower deductible plan. Future research should examine HDHP enrolment among members who have plan choice to better understand factors associated with selecting HDHPs.

\section{CONCLUSION}

HDHP enrolment has increased rapidly among both healthy and chronically ill populations and by 2013 over half of members with chronic conditions in one large national insurer had HDHPs. HDHP members pay significantly more OOP for their healthcare than low deductible plan members, and HDHP members with chronic diseases have OOP spending that is five to seven times higher than that of healthy members. Policymakers should consider options for protecting clinically vulnerable patients enrolled in HDHPs, such as funding HSAs or facilitating enrolment in more generous plan designs. ${ }^{26}$

\section{Twitter Laura F Garabedian @LFGarabedian}

Contributors LFG, DRD and JFW contributed to the conception and design of the project. LFG, FZ, RL, JW, DRD and JFW contributed to the acquisition, analysis and interpretation of the data. LFG, FZ, RL, JW, DRD and JFW contributed to drafts of the manuscript. LFG provided final approval of the version to be published and is accountable for the accuracy and integrity of the work.

Funding This work was supported by grants from Centers for Disease Control (CDC) and Prevention/National Institute of Diabetes and Digestive and Kidney Diseases (NIDDK) (5U18DP006122) and the NIDDK Health Delivery Systems Centre for Diabetes Translational Research (1P30-DK092924). The content is solely the responsibility of the authors and does not necessarily represent the official views of the National Institutes of Health or the US CDC.

Competing interests None declared.

Patient consent for publication Not required.

Ethics approval This study was approved by the Harvard Pilgrim Health Care Institutional Review Board.

Provenance and peer review Not commissioned; externally peer-reviewed.

Data availability statement Data may be obtained from a third party, but are not publicly available. Data are not shareable because of our data use agreement with the data vendor, but we are happy to share programming code upon request.

Supplemental material This content has been supplied by the author(s). It has not been vetted by BMJ Publishing Group Limited (BMJ) and may not have been peer-reviewed. Any opinions or recommendations discussed are solely those of the author(s) and are not endorsed by BMJ. BMJ disclaims all liability and responsibility arising from any reliance placed on the content. Where the content includes any translated material, BMJ does not warrant the accuracy and reliability of the translations (including but not limited to local regulations, clinical guidelines, terminology, drug names and drug dosages), and is not responsible for any error and/or omissions arising from translation and adaptation or otherwise.

Open access This is an open access article distributed in accordance with the Creative Commons Attribution Non Commercial (CC BY-NC 4.0) license, which permits others to distribute, remix, adapt, build upon this work non-commercially, and license their derivative works on different terms, provided the original work is properly cited, appropriate credit is given, any changes made indicated, and the use is non-commercial. See: http://creativecommons.org/licenses/by-nc/4.0/.

ORCID iD

Laura F Garabedian http://orcid.org/0000-0002-0014-4181

\section{REFERENCES}

1 The Kaiser Family Foundation and Health Research \& Educational Trust. Employer health BenefitsL 2017 annual survey. Menlo Park, California: The Kaiser Family Foundation and Health Research \& Educational Trust, 2017.

2 The Kaiser Family Foundation. Employer health benefits 2019 annual survey San Francisco. California: The Kaiser Family Foundation, 2019.

3 Beeuwkes Buntin M, Haviland AM, McDevitt R. Healthcare spending and preventive care in high-deductible and consumer-directed health plans. Am J Manag Care 2011;17:222-30.

4 Frostin P, Dretzka E. Consumer Engagement in Health Care: Findings From the 2018 EBRI/Greenwald \& Associates Consumer Engagement in Health Care Survey. EBRI Issue Brief, no. 468. Employee Benefit Research Institute. Available: https://www.ebri. org/crawler/view/high-deductible-health-plan-enrollees-are-moreengaged-in-their-health-care

5 Lave JR, Men A, Day BT, et al. Employee choice of a highdeductible health plan across multiple employers. Health Serv Res 2011;46:138-54.

6 Anon. Intensive blood-glucose control with sulphonylureas or insulin compared with conventional treatment and risk of complications in 
patients with type 2 diabetes (UKPDS 33). UK prospective diabetes study (UKPDS) group. Lancet 1998;352:837-53.

7 Holman RR, Paul SK, Bethel MA, et al. 10-year follow-up of intensive glucose control in type 2 diabetes. N Engl J Med 2008;359:1577-89.

8 Capewell S, Morrison CE, McMurray JJ. Contribution of modern cardiovascular treatment and risk factor changes to the decline in coronary heart disease mortality in Scotland between 1975 and 1994. Heart 1999;81:380-6.

9 Anon. Standards of medical care in diabetes--2015: summary of revisions. Diabetes Care 2015;38 Suppl:S4.

10 American Diabetes Association. Standards of Medical Care in Diabetes-2019 Abridged for Primary Care Providers. Clin Diabetes 2019;37:11-34.

11 Centers for Disease Control and Prevention. Get active! Atlanta, GA: Centers for Disease Control and Prevention, 2018. https://www.cdc. gov/diabetes/managing/active.html

12 American Diabetes Association. 10. Cardiovascular Disease and Risk Management: Standards of Medical Care in Diabetes-2020. Diabetes Care 2020;43:S111-34.

13 American College of Cardiology. Guidelines and clinical documents: American College of cardiology, 2020. Available: https://www.acc. org/guidelines [Accessed 30 June 2020].

14 Wharam JF, Lu CY, Zhang F, et al. High-Deductible insurance and delay in care for the macrovascular complications of diabetes. Ann Intern Med 2018;169:845-54.

15 Wharam JF, Zhang F, Eggleston EM, et al. Diabetes outpatient care and acute complications before and after high-deductible insurance enrollment: a natural experiment for translation in diabetes (NEXT-D) study. JAMA Intern Med 2017;177:358-68.

16 Wharam JF, Zhang F, Eggleston EM, et al. Effect of high-deductible insurance on High-Acuity outcomes in diabetes: a natural experiment for translation in diabetes (NEXT-D) study. Diabetes Care 2018;41:940-8.
17 Lewey J, Gagne JJ, Franklin J, et al. Impact of high Deductible health plans on cardiovascular medication adherence and health disparities. Circ Cardiovasc Qual Outcomes 2018;11:e004632.

18 Reid RJ, Roos NP, MacWilliam L, et al. Assessing population health care need using a claims-based ACG morbidity measure: a validation analysis in the Province of Manitoba. Health Serv Res 2002;37:1345-64.

19 The Johns Hopkins University. The Johns Hopkins ACG system. Baltimore, MD: The Johns Hopkins University, 2020. https://www. hopkinsacg.org/advantage/

20 United States Census Bureau. American community survey (ACS). Washington, D.C: United States Census Bureau, 2020. https://www. census.gov/programs-surveys/acs/

21 STATA. Marginal means, adjusted predictions, and marginal effects. Available: https://www.stata.com/features/overview/marginalanalysis/ [Accessed 27 Jan 2021].

22 Williams R. Using the margins command to estimate and interpret adjusted predictions and marginal effects. Stata J 2012;12:308-31.

23 Desilver D. For most U.S. workers, real wages have barely budged in decades. Washington, D.C: Pew Research Center, 2018. https:// www.pewresearch.org/fact-tank/2018/08/07/for-most-us-workersreal-wages-have-barely-budged-for-decades/

24 Kullgren JT, Cliff EQ, Krenz C, et al. Use of health savings accounts among US adults enrolled in high-deductible health plans. JAMA Netw Open 2020;3:e2011014.

25 Fronstin P, Roebuck MC. Persistency in high-cost health care claims: "It's where the spending is, stupid." EBRI Issue Brief, no. 493. Employee Benefit Research Institute, 2019. Available: https://www. ebri.org/crawler/view/persistency-in-high-cost-health-care-claims-its-where-the-spending-is-stupid

26 Wharam JF, Ross-Degnan D, Rosenthal MB. The ACA and highdeductible insurance--strategies for sharpening a blunt instrument. $N$ Engl J Med 2013;369:1481-4 